\title{
Mindestmengen in der Hüftendoprothetik - Qualität durch Quantität?
}

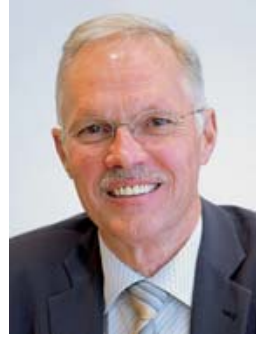

F. U. Niethard

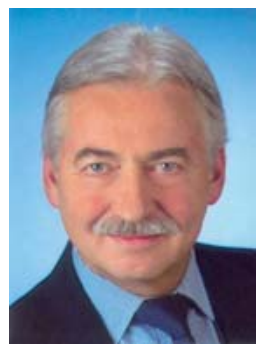

K. Weise

Bibliografie

DOI 10.1055/s-2007-965388 Z Orthop Unfallchir 2007; 145: 261-262 @ Georg Thieme Verlag KG Stuttgart • New York . ISSN 1864-6697

\section{Korrespondenzadresse} Prof. Dr. med. Fritz U. Niethard Orthopädische Klinik Universitätsklinik der RWTH Aachen Pauwelsstraße 30 52074 Aachen Tel.: 0241/808-9410 Fax: 0241/808-2453 funiethard@ orthopaedie-aachen.de

Prof. Dr. med. Kuno Weise BG-Unfallklinik

Schnarrenbergstraße 95 72076 Tübingen

Tel.: $07071 / 606-1001$

Fax: 07071/606-1002 weise@bgu-tuebingen.de
Der Gesetzgeber hat in $§ 137$ Absatz 1 Nr. 3 SGB V festgelegt, dass für „planbare Leistungen, bei denen die Qualität des Behandlungsergebnisses in besonderem Maße von der Menge der erbrachten Leistungen abhängig ist, Mindestmengen je Arzt oder Krankenhaus“ festgelegt werden können. Die Bestimmung im SGB V, wonach zwischen Ergebnisqualität und Leistungsmenge das Verhältnis einer Abhängigkeit bestehen muss, wird in der sog. Mindestmengenvereinbarung zwischen den Spitzenverbänden der Krankenkassen, dem Verband der Privaten Krankenversicherungen und der Deutschen Krankenhausgesellschaft im Einvernehmen mit der Bundesärztekammer und dem Deutschen Pflegerat dahingehend präzisiert, dass zwischen beiden Parametern ein kausaler Zusammenhang nachgewiesen sein muss. Eine bloße statistische Assoziation wäre demnach nicht ausreichend [3].

Der gemeinsame Bundesausschuss hat diesen kausalen Zusammenhang zwischen Menge und Qualität für die Knieendoprothetik anerkannt und eine verbindliche Mindestmenge von 50 Eingriffen pro Jahr pro Krankenhaus oder Arzt ab dem 1. Januar 2006 beschlossen.

Die Entscheidungen zu Mindestmengenregelungen im Allgemeinen und zur Knieendoprothetik im Speziellen haben zu zahlreichen Diskussionen geführt. Die Zielvorstellungen des Gesetzgebers sind eindeutig: Neben einer Qualitätsverbesserung wird natürlich auch ein wirtschaftlicher Konzentrationsprozess angestrebt. Trotz der bisher geringen Anzahl von Prozeduren, die der Mindestmengenregelung unterliegen, kann dies im Einzelfall zur ernsthaften Bedrohung von Krankenhäusern führen.

Die Mindestmengenregelung im Allgemeinen wurde ausführlich anlässlich des 107. Deutschen Ärztetages in Bremen diskutiert. In dem von der Bundesärztekammer in Auftrag gegebenen Gutachten von Geraedts konnte nur eine spärliche Evidenz für explizite Mindestmengen nachgewiesen werden [2]. Für eine Mindestmengenregelung spräche, dass

- die Versorgungsergebnisse der Patienten besser würden,

- die Fallkosten geringer werden,

- spezialisierte Krankenhäuser attraktiver werden für spezialisierte Ärzte und Kompetenzzentren entstehen,

- die Patienten in diesen Einrichtungen auch ein in anderer Hinsicht positiveres Behandlungsumfeld erfahren könnten.
Gegen die Einführung von Mindestmengen wird angeführt, dass

- Leistungserbringer mit geringer Fallzahl aber hoher Versorgungsqualität ausgeschlossen werden,

- die flächendeckende Versorgung der Patienten eingeschränkt wird,

- die Erfahrungen der behandelnden Ärzte eingeschränkt werden,

- die Kontinuität der Behandlung eingeschränkt wird, weil diese nur noch in Zentren möglich ist,

- bewährte Netzwerke zerstört werden,

- bei Umschichtungsprozessen Wartelisten entstehen,

- die ärztliche Weiterbildung erschwert wird,

- Fehlanreize für eine breitere Indikationsstellung entstehen können,

- das Bemühen um Qualitätsverbesserung eingeschränkt würde,

- die Krankenhauspläne durch die Mindestmengenregelungen umgeschrieben werden müssten.

Der Ärztetag kam daher zu dem Schluss, dass „exakte Mindestmengen als Qualitätsindikatoren aus Studien nicht evidenzbasiert ableitbar seien. Der Begriff der Mindestmenge sei am ehesten geeignet, ökonomische Anforderungen zu beschreiben“. Der Deutsche Ärztetag forderte die Politik auf, „die Entwicklung von Qualitätsindikatoren und eine begleitende Evaluierungsforschung zum Zusammenhang zwischen Leistungsfrequenz und Ergebnisqualität durch Ressourcenbereitstellung zu unterstützen“.

Auch die wissenschaftlichen Gesellschaften von Orthopädie (DGOOC) und Unfallchirurgie (DGU) haben sich zur Einführung der Mindestmengenregelung für die Knieendoprothetik geäußert. Wenngleich dabei ebenfalls auf die gleiche Literatur zurückgegriffen wurde, war der Tenor der Schlussfolgerung doch unterschiedlich. Das von der DGOOC vorgelegte Gutachten hielt die Mindestmengenregelung für den Bereich Knieendoprothetik für gut begründbar, während von Seiten der DGU die Evidenzlage als nicht ausreichend bezeichnet wurde.

Es war zu erwarten, dass in Anbetracht der durch die demografische Entwicklung stark steigenden Zahl von Endoprothesenoperationen nun auch die Hüftendoprothetik in diese Diskussion einbezogen wird. Smektala et al. [6] haben bereits 2005 die Daten der externen Qualitätssicherung in Nordrhein-Westfalen in dieser Hinsicht überprüft. Sie kamen zu dem Schluss, „dass sich ein Schwellenwert für die Endoprothetik des Hüft- 
gelenkes aus den BQS-Daten für das Land Nordrhein-Westfalen nicht festlegen lässt“. Dieses Heft der „Zeitschrift für Orthopädie und Unfallchirurgie“" wird durch zwei weitere Arbeiten zu diesem Thema eingeleitet $[4,5]$, mit denen die Herausgeber Informationen zu dieser Thematik vertiefen wollen. Beide Arbeiten stützen sich in ihren Aussagen auf die Auswertung medizinischer Datenbanken. Koy et al. untersuchen vorrangig den Einfluss der Mindestmengen auf die Ergebnisqualität in der Hüftendoprothetik, während sich Schräder und Rath darüber hinaus auch mit den Auswirkungen auf die flächendeckende Versorgung beschäftigen. Interessant ist, dass von den zahlreich zu dieser Thematik erschienenen Studien jeweils nur 17 bzw. 13 Arbeiten in die Auswertung eingeschlossen werden konnten, weil sie sich differenziert mit der „Volume-Outcome-Beziehung“ befasst haben.

Der Vergleich beider Arbeiten hinsichtlich des Zusammenhanges von Mindestmengen und Ergebnisqualität zeigt, dass immer noch Interpretationsspielraum besteht. Koy et al. kommen zu dem Schluss, „dass ein eindeutiger Trend feststellbar ist, nachdem das Operationsergebnis nach primärer Hüft-TEP-Implantation sowie nach Revisions-Hüft-TEP-Implantation durch Krankenhäuser mit hoher Fallzahl und vor allem durch Chirurgen mit hoher Fallzahl verbessert werden kann. Je nachdem, welche Schwellenwerte man für ein hohes und ein niedriges Operationsvolumen einsetzt, können diese Ergebnisse auch statistische Signifikanz erreichen“. Auch Schräder und Rath konnten in 11 der von ihnen untersuchten 13 Studien einen positiven $\mathrm{Zu}$ sammenhang zwischen höherer Behandlungsmenge pro Krankenhaus und/oder Arzt und besserer Behandlungsqualität feststellen. Einen einheitlichen Grenzwert konnten sie jedoch nicht ermitteln. Die Schwellenwerte lagen zwischen 2 Endoprothesen pro Jahr pro Arzt und 130 Hüftendoprothesen pro Jahr pro Krankenhaus. Dies zeigt, dass der Bezug der Mindestmengenregelung auf Krankenhäuser zu einer statistischen Fehlerbreite führen muss; denn der Zusammenhang zwischen Quantität und Qualität wird deutlicher, wenn die Ergebnisse auf den einzelnen Arzt oder die Abteilung bezogen werden. Durch die Zusammenfassung von Krankenhäusern in Verbünden oder von Abteilungen in Kliniken gehen diese Zusammenhänge verloren.

Beide Autorengruppen sind sich daher der limitierten Aussagekraft ihrer Daten bewusst, denn entsprechende Untersuchungen zur Versorgung liegen in Deutschland nicht vor. Koy et al. regen daher an, dass bedachtsam über anstehende Veränderungen diskutiert werden müsse, um weitere Verbesserungen zu erreichen.

Zu diesen Veränderungen nehmen Schräder und Rath Stellung, indem sie die Auswirkungen gesondert für die Versorgung bei Coxarthrose und Schenkelhalsfraktur untersuchen. Nach ihrer Analyse wurden in Deutschland 2004 in 1264 Abteilungen ca. 150000 Hüftendoprothesen implantiert, davon ca. 120000 bei
Coxarthrose und 30000 bei Schenkelhalsfraktur. In vielen Krankenhäusern werden immer noch sehr wenige Hüftendoprothesen pro Jahr eingesetzt, in immerhin $16 \%$ aller Kliniken weniger als 20 Fälle pro Jahr. Die Einführung einer Mindestmengenregelung bei der Hüftendoprothetik müsste natürlich ebenso wie bei der Knieendoprothetik zu einem Umverteilungsprozess führen. Eine selektive Mindestmenge von 50 Operationen pro Jahr würde für die elektive Hüftendoprothetik 553 Krankenhäuser (48\%) betreffen und annähernd 11000 Patienten (9,7\%) müssten umverteilt werden. Dies ist in etwa die Größenordnung, die auch für die Knieendoprothetik kalkuliert wurde [1]. Problematisch wird es dagegen für die primäre Hüftendoprothetik bei Schenkelhalsfraktur. Eine Mindestmengenregelung von 50 würde 1006 Krankenhäuser $(85,1 \%)$ betreffen und 58,5\% aller Patienten müssten umverteilt werden. Demnach wäre eine Regelung nur als prozedurenbezogene aber nicht indikationsbezogene Mindestmenge festzulegen, weil nur diese „keinen unkalkulierbaren negativen Einfluss auf die flächendeckende Versorgung in Deutschland haben würde“. Die Zusammenführung von Orthopädie und Unfallchirurgie könnte hier die so häufig beschworenen Synergien geltend machen. Diese Zusammenführung ist aber für die flächendeckende Versorgung bei weitem noch nicht abgeschlossen.

Aus diesem Grund kann den Autoren nur zugestimmt werden, wenn über die anstehenden Veränderungen bedachtsam diskutiert bzw. der Schwellenwert mit Augenmaß und unter Berücksichtigung der Versorgungssituation in Deutschland festgesetzt werden muss. Die wissenschaftlichen Gesellschaften DGOC und DGU sind zu dieser Thematik erneut gefragt.

\section{F. U. Niethard, Aachen}

K. Weise, Tübingen

\section{Literatur}

1 Blum K, Offermanns M. Mindestmengenregelung - Wirkung auf Versorgungsstruktur und Patientenwanderung. Arzt und Krankenhaus 2004; $11: 337-341$

2 Geraedts $M$. Spärliche Evidenz für explizite Mindestmengen. Deutsches Ärzteblatt 2004; 101: A14/02 - A14/04

3 Klakow-Franck R, Wetzel H. Krankenhäuser/Qualitätssicherung: Mindestmengenregelung ohne Mindestmengen. Deutsches Ärzteblatt 2004; 101: A2926-A2928

4 Koy T, König DP, Eysel P. Einfluss von Mindestmengen auf die Ergebnisqualität in der Hüftendoprothetik. Z Orthop Unfallchir 2007; 145: $291-296$

5 Schräder P, Rath T. Mindestmengen in der Hüftgelenksendoprothetik bei Coxarthrose und Schenkelhalsfraktur - Evidenzbericht und Modellrechnung zur Auswirkung auf die flächendeckende Versorgung. Z Orthop Unfallchir 2007; 145: 281 - 290

6 Smektala R, Schultze-Raestrup U., Wittenberg R., Bredehöft J. Mindestmengen in der Hüftgelenk-Endoprothetik bei Coxarthrose. Z Orthop 2005; 143: 4-7 